\title{
A new perspective for nonphotochemical quenching in plant antenna complexes
}

\author{
Edoardo Cignoni * $\quad$ Margherita Lapillo* Lorenzo Cupellini*† \\ Silvia Acosta-Gutiérrez ${ }^{\ddagger} \quad$ Francesco Luigi Gervasio $\$ \mathbf{I}$ \\ Benedetta Mennucci*\|
}

\begin{abstract}
Light-harvesting complexes (LHCs) of plants exert a dual function of light-harvesting and photoprotection. While LH processes are relatively well characterized, those involved in photoprotection are less understood. The main mechanism involved in photoprotection is to dissipate the energy absorbed by chlorophylls into harmless heat through processes collectively called nonphotochemical quenching (NPQ). Here, we characterize the quenching mechanisms of CP29, a minor LHC of plants with an important role in photoprotection, through two complementary enhanced-sampling techniques, dimensionality reduction schemes, electronic calculations and the analysis of cryo-EM data in the light of the predicted conformational ensemble. Our analysis reveals that the mechanism is more complex than previously thought. Several conformations of the lumenal side of the protein occur and differently affect the pigments relative geometries and interactions. Moreover, we show that a quenching mechanism localized on a single pair of pigments is not sufficient but many pigments are simultaneously involved. In such a diffuse mechanism, short-range interactions between each carotenoid and different chlorophylls combined with a protein-mediated tuning of the carotenoid excitation energies, have to be considered in addition to the commonly suggested coulomb interactions.
\end{abstract}

\section{Introduction}

Light-harvesting complexes (LHCs) in the photosystems of plants and green algae use aggregates of chlorophylls (Chls) to collect sunlight and transfer excitation energy to the photosystem core. However, an excess of light can be detrimental to such a delicate photosynthetic apparatus, and photoprotective mechanisms are needed to avoid photodamage ${ }^{1,2}$. The main strategy is to dissipate the energy absorbed by chlorophylls into harmless heat; this is made possible through processes collectively called nonphotochemical quenching (NPQ) ${ }^{3-8}$.

The ways through which such a dissipation proceeds is still debated. Most authors agree that the carotenoid molecules (Cars) embedded in LHCs actively participate in the quenching ${ }^{9-11}$. Within this hypothesis, the most widely accepted mechanism for the quenching involves excitation energy transfer (EET) from the excited Chls to the dark $\left(\mathrm{S}_{1}\right)$ state of Cars ${ }^{9,12}$. A second mechanism has also been proposed which entails a charge transfer (CT), whereby the excited Chls accept an electron from the Cars ${ }^{10,11,13,14}$.

\footnotetext{
* Dipartimento di Chimica e Chimica Industriale, University of Pisa, via G. Moruzzi 13, 56124, Pisa, Italy

${ }^{\dagger}$ Corresponding author: lorenzo.cupellini@unipi.it

${ }^{\ddagger}$ Department of Chemistry, University College London, WC1E 6BT London, UK

$\S$ ISPSO, University of Geneva, CH-1211 Geneva, Switzerland

$\mathbb{I}_{\text {Corresponding author: francesco.gervasio@unige.ch }}$

\| Corresponding author: benedetta.mennucci@unipi.it
} 
Recent experimental studies also suggest that these quenching processes are mediated by conformational changes of the LHCs ${ }^{15-19}$. As a matter of fact, LHCs can access various conformational states that present specific spectral and kinetic properties, but their connection to quenched and unquenched states are still to be revealed ${ }^{16,20-22}$. Moreover, while it is clear that NPQ is triggered by a $\mathrm{pH}$ decrease in the lumenal side of the membrane and the following activation of an additional protein, the PsbS protein ${ }^{2,22}$, the mechanisms through which PsbS interact with the LHC and affects its state are not known.

In this work, we focus on a minor LHC of higher plants and green algae, CP29 (Figure 1). At variance with the main antenna complex, LHCII, CP29 is a monomeric complex, found at the interface between the peripheral antenna complexes and the core complexes of Photosystem II. Its central position makes CP29 an ideal site for photoprotection ${ }^{23}$. CP29 binds 13-14 chlorophylls and three carotenoids (Figure 1b), namely lutein (Lut), violaxanthin (Vio) and Neoxanthin $(\mathrm{Neo})^{24,25}$. The binding sites of Lut (L1) and Vio (L2) have both been related to the photoprotective function ${ }^{19,26,27}$. In particular, it is thought that Lut and Vio can quench the excitation of the tightly associated Chls a 612 and $a 603^{28}$, respectively. Both of them have been related to an excitation-energy sink, the "terminal emitter" domain ${ }^{9,19,29}$. In all cases the quenching, is believed to go through an excitation energy transfer which is determined, according to the Förster theory, by the Coulomb interactions between excitations localized on the Chl and the Car, respectively. Within this model, in order to shift from the active to the quenched state, a significant change in Chl-Car interactions (e.g. in the value of their coulomb coupling) is necessary. This, in turn, implies a large modification of the relative distance and/or orientation of the two interacting pigments which should follow the conformational change of the complex.

Here a different perspective is suggested for the regulation of NPQ activity in CP29. By combining two complementary enhanced sampling techniques ${ }^{30,31}$ with unbiased molecular dynamics simulations ${ }^{32,33}$ and dimensionality reduction methods, we thoroughly explore the conformational landscape of the complex and establish how the apoprotein conformation impacts the L1 and L2 sites and the interactions among the embedded pigments. These effects are quantified in terms of both long-range Coulomb couplings and short-range effects. Our analysis reveals that the hypothesis of a switch between an active and a quenched state is too simplistic, and instead several conformations of the lumenal side of the protein occur and differently affect the pigments relative geometries and interactions. Moreover, we show that a description based solely on the Coulomb interactions in the most coupled Car-Chl pairs cannot explain the photoregulation. Instead, a more diffuse mechanism involving all the pigments within both L1 and L2 sites is possible. In such a mechanism, short-range interactions between each carotenoid and different Chls, combined with a protein-mediated tuning of the carotenoid excitation energies, have to be considered.

\section{Results and Discussion}

\section{Exploration of protein conformations}

The conformational sampling of CP29 is here enhanced by employing Parallel Tempering in the Well-Tempered Ensemble (PT-WTE) ${ }^{34,35}$. This approach, which was successfully used in a number of systems ${ }^{36,37}$, leads to a faster exploration of the conformational landscape of the CP29 complex. In this way, free energy barriers of moderate size can be overcome without explicitly introducing a collective coordinate to bias the protein structure or the pigments towards specific conformations. Details on the simulation protocol are provided in the SI. Increasing evidence ${ }^{4,17,21}$ indicates that LHCs possess a highly heterogeneous conformational ensemble, whose equilibrium can be tuned by PsbS, the establishment of a $\mathrm{pH}$ gradient, and aggregation ${ }^{20,22}$. To capture and disentangle the conformational heterogeneity of CP29 we apply a Principal Component Analysis (PCA) on the backbone and sidechain dihedrals $\left(\mathrm{dPCA}^{38}\right)$ of the protein residues. The low-dimensional projection of the CP29 dynamics onto the first two dPCA principal components is shown in Figure 2, and compared with a previous unbiased simulation of CP29 (cMD $\mathrm{CryoEM})^{39}$. From this comparison, 
a

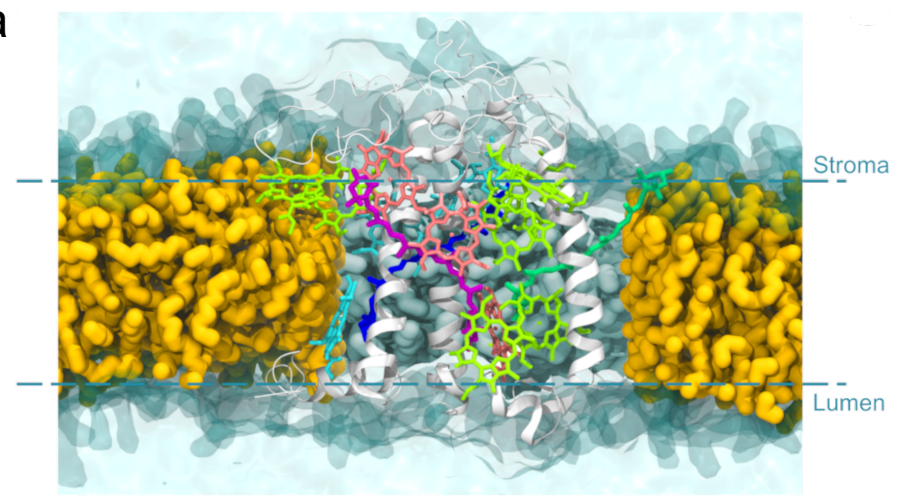

b

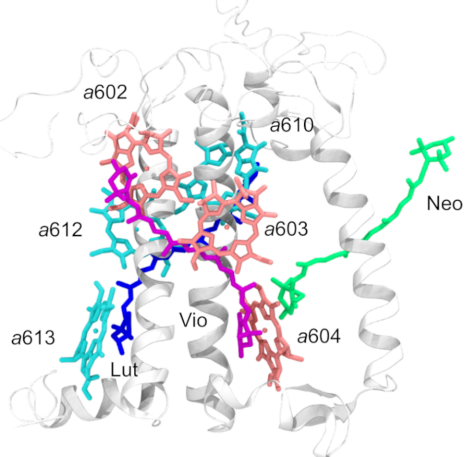

C
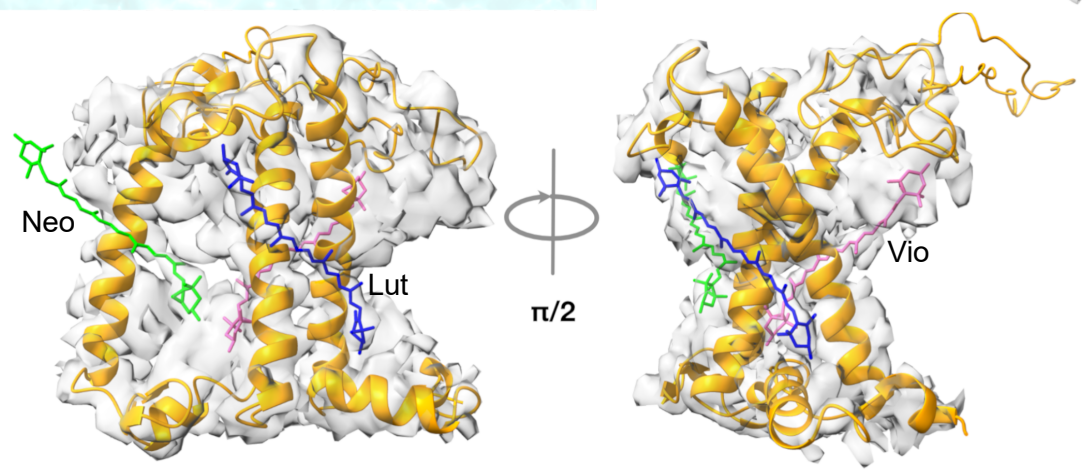

Figure 1: The CP29 antenna complex. (a) CP29 embedded in the phospholipid membrane employed in our simulations. The lipid membrane is cut for a better visualization of the complex. (b) The carotenoids and some of the chlorophylls embedded in CP29: Lut (blue) in L1, Vio (purple) in L2, and Neo (green) in N1. Only the Chls in sites L1 and L2 are shown and highlighted in cyan and pink, respectively. (c) CP29 cryo-EM structure (PDB: 3JCU) embedded in the cryo-EM electron density.

it is clear that the PT-WTE enhanced sampling approach explores a larger portion of conformational space, in both the stromal and lumenal sides of the complex.

The enhanced conformational freedom of the stromal part of the protein (Figure 2) arises from the high degree of flexibility of the N-terminal domain of the protein, which protrudes outside the membrane in our simulations (Figure 1a). This observation is in line with previous reports. ${ }^{24,40}$ Indeed, the first 87 residues in the $\mathrm{N}$-terminal domain outside the membrane are so flexible in the isolated CP29 that their crystal structure could not be determined. ${ }^{24}$ The situation is different when the CP29 is embedded in the much larger biological PSII complex as the N-terminal domain anchors the CP29 to CP47. In this case, the flexibility of the region is significantly less pronounced, as shown by the cryo-EM electron density (see also below). ${ }^{41,42}$

Conversely, the observed enhanced plasticity of the lumenal side of the protein should not be affected by the contacts of CP29 in the PSII and it is in line with previous suggestions that the conformational changes triggering the onset of quenched conformations take place on this side of the LHCs ${ }^{43-45}$. As discussed below, a marked flexibility of this side of the protein is also observed in the electron density of the whole PSII complex obtained by cryo-EM. ${ }^{41}$ Thus, we first focus our analysis on the lumenal side of the complex, and in particular on the region surrounding helix D.

The PT-WTE lumenal free energy landscape is characterized by different minima exhibiting similar stability (Figure 2a) on the projection variables. The broadest one contains the structure reconstructed from cryo-EM data (PDB:3JCU ${ }^{41}$ ) and crystallography ${ }^{24}$. In order to better characterize protein conformations, we have grouped similar structures together with a hierarchical clustering algorithm. We have identified six clusters (Figure $2 \mathrm{~b}, \mathrm{~d}$ ), corresponding to six free en- 
ergy minima in the dPCA space. Details on the clustering procedure and on the selection of the clusters are provided in the SI.

a

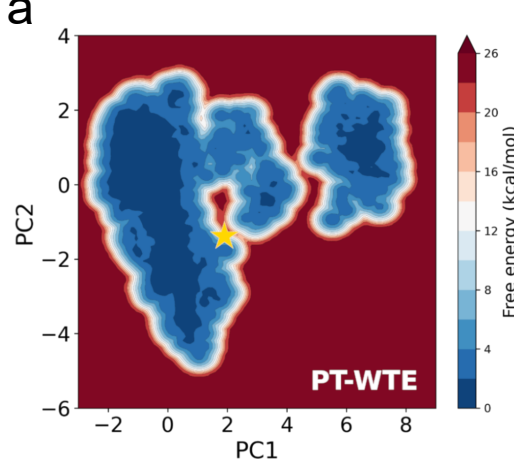

b

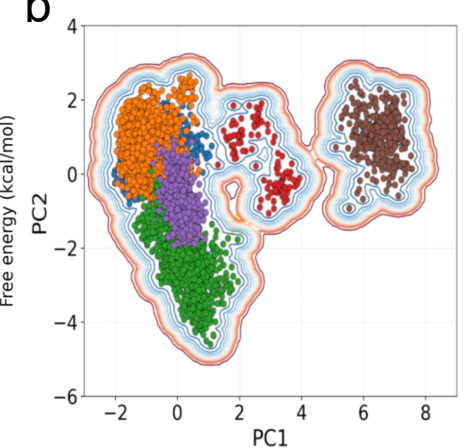

C

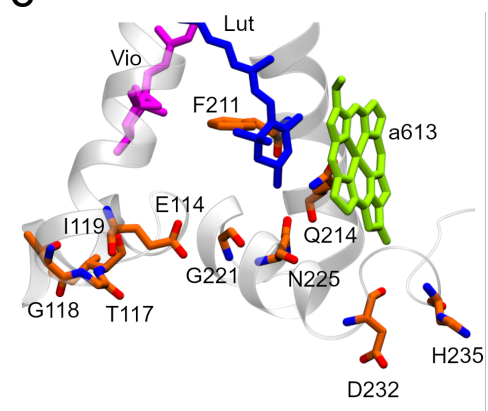

d

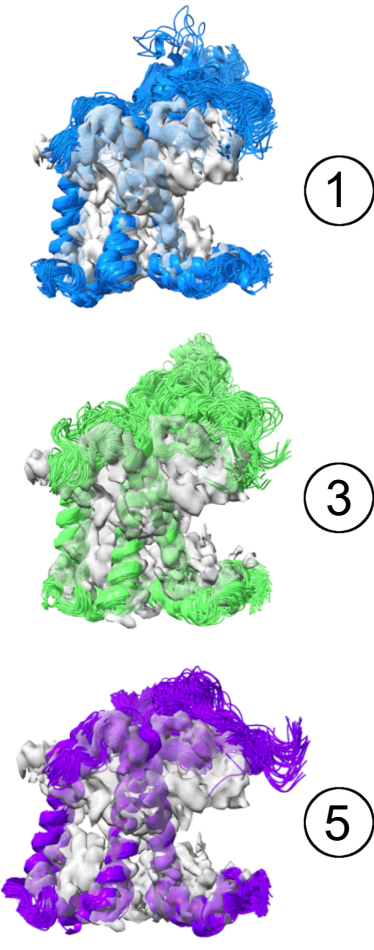

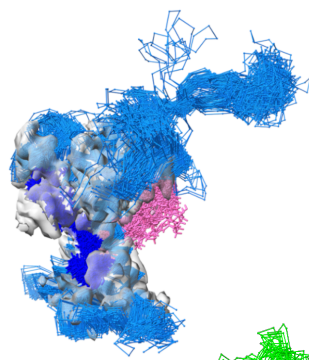

(3)
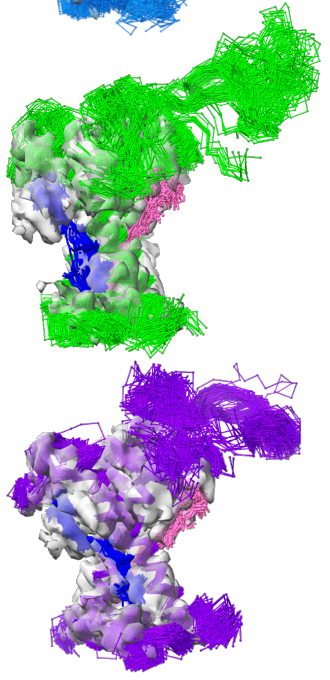

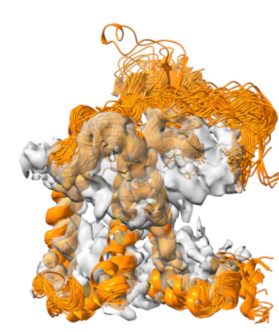

2
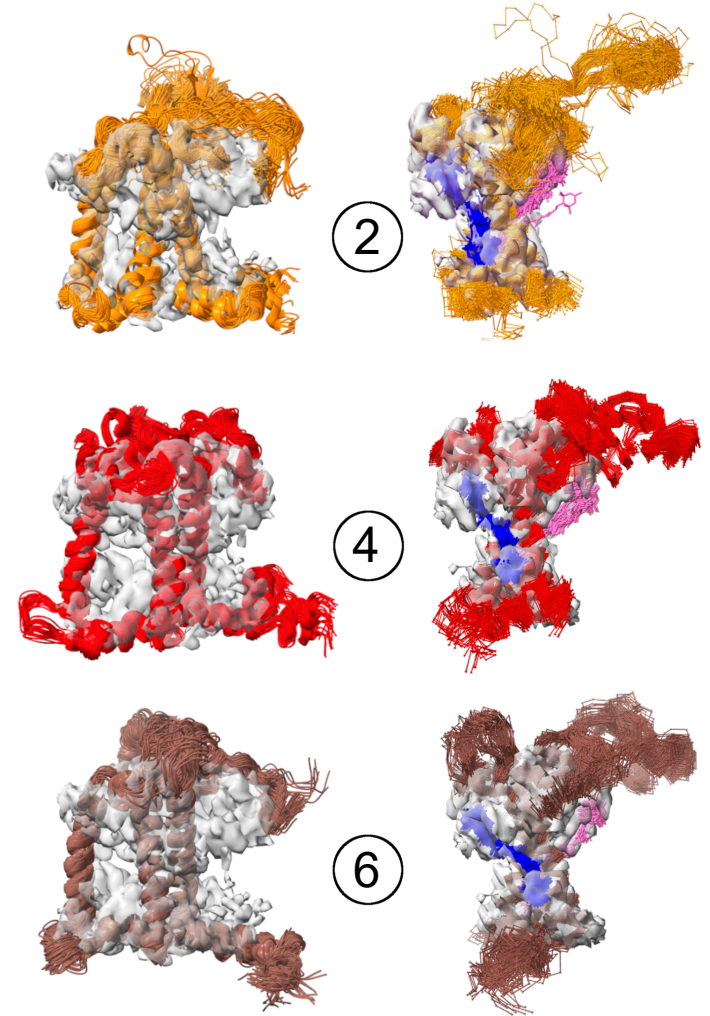

Figure 2: Conformational landscape of CP29 explored in the PT-WTE simulation. (a) Exploration of the PT-WTE simulations of CP29, shown in the space of the first two dPCA principal components. The position of the cryo-EM starting structure (PDB: 3JCU) is shown as a yellow star. (b) Clustering of the PT-WTE simulation, visualized in APCA space. Points colored differently correspond to different clusters. (c) Most relevant residues of the dynamics of CP29 on its lumenal side (see text). The carotenoids are shown for reference, as well as Chl a613 whose axial ligand is Q214. (d) Visualization of the structures sampled in the PT-WTE simulation. The structures are fitted in the cryo-EM density so as to better visualize their difference with the starting structure. The ID of the cluster is indicated in each circle.

Figure $2 \mathrm{~b}$ shows the distribution of the clusters in the first two dimensions of the dPCA space, and in Figure $2 \mathrm{~d}$ random samples of a few structures per cluster are shown as cartoon with the cryo-EM ${ }^{41}$ electron density overlaid for comparison. Clusters 4 and 6 correspond to the two well- 
separated minima in the free energy surface (Figure 2a), and show significant differences in the protein backbone at the lumenal side. The remaining clusters are closer together, and show similar backbone structures (Figure 2d). Nonetheless, these clusters can be distinguished upon examination of the third PC (Figure 4) and by looking at the distributions of their sidechain conformations (Figure 5a).

The most striking difference observed in clusters 4 and 6 is represented by an alternative position and orientation of helix D. Indeed, compared to the other helices, helix D presents the highest B-factor in the cryo-EM data ${ }^{41}$ (Figure 7). Furthermore, the main helices are very well defined in the electron density, including the N-terminal of the complex, but the C-terminal is less well resolved (Figure 1c), suggesting that this helix was present in multiple orientations in the cryo-EM dataset used for the structure reconstruction. In agreement with the cryo-EM data, we observe a high mobility of helix D, which sticks out from the electron density in all the clusters. In addition, in clusters 4 and 6 helix D is in a markedly different position, farther from from helix E and slightly shifted towards the lumen (Figure 6). In agreement with Ioannidis et al. ${ }^{44}$, we find that the displacement of helix D observed in clusters 4 and 6 is associated with a different backbone geometry of G221. This enhanced flexibility is also in line with the findings of Ref. ${ }^{46}$, where an expansion of the lumenal side of the complex was observed for LHCII monomers, as opposed to the trimeric LHCII.

In addition to G221, several other amino acids are found to be important in determining the identity of the different protein conformations explored by our simulation (Figure 3), and some of them are shown in Figure 2c. They are all localized on the lumenal side of the protein, with the exception of F221 and Q214. Interestingly, Q214 is the axial ligand of Chl a613, while F211 is localized near the lumenal ring of Lut, suggesting that conformational changes at the level of the protein residues do occur at the level of the L1 pocket, as recently suggested ${ }^{47}$. In addition, several residues (G221, N225, D232, H235) appear to play a role in determining the orientation and the geometry of helix D and the C-terminus. Finally, some residues (E114, T117, G118, I119) are localized on the lumenal side of helix B and on the loop that links helix B to helix E, possibly affecting the geometry of helix E and the shape of the L2 pocket.

\section{Protein conformation affects pigment geometries and interactions}

Given the conformational changes observed in the PT-WTE simulation, it is of interest to see if they do impact the L1 and L2 sites and the embededd pigments. It has been reported that the pigments' interactions are extremely sensible to an alteration of their environment ${ }^{48,49}$, both at the level of the relative intermolecular arrangement and at the level of the pigments' internal geometries. The latter point is especially subtle for carotenoids ${ }^{45}$, for which minor changes in the geometry can significantly influence their electronic structure and, indirectly, the quenching processes ${ }^{50,51}$. Indeed, our simulations show that carotenoid conformations differing in their conjugated chain geometry are present in CP29 (Figure 8), in agreement with the results of Liguori et al. on LHCII ${ }^{45}$. The most pronounced changes are observed for the first conjugate chain dihedrals on the lumenal side of Lut and the stromal side of Vio, so that the carotenoids can shift between s-cis and strans conformations via a pedaling mechanism (Figure 8). Furthermore, we observe that different dihedral distributions are associated with different clusters, supporting the view that the protein scaffold directly contributes to the establishment of different carotenoid geometries in its L1 and L2 pockets. As variations in the carotenoid conjugated chain are expected to have an effect on its excited state properties, this suggests that small conformational changes at the level of the L1 and L2 pockets can tune the $S_{1}$ energies of the embedded carotenoids, as previously proposed ${ }^{39,45}$, resulting in a modulation of the energy transfer processes mediated by the protein scaffold.

In addition to internal conformational changes of the carotenoids, the quenching pathways strongly depend on Car-Chl interactions. The EET quenching by Car $\mathrm{S}_{1}$ states has been understood in the framework of Förster theory, where the energy transfer rate depends on the squared electronic coupling and the spectral overlap between Chl emission and Car absorption. Moreover, 
a
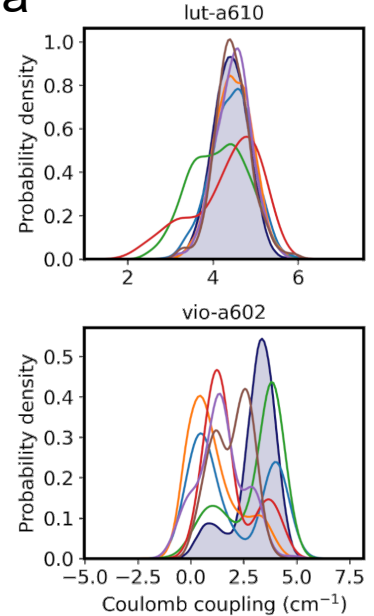

d

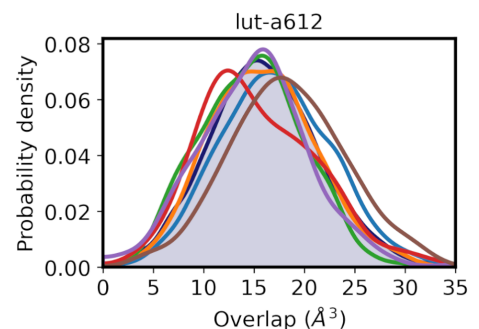

lut-a612

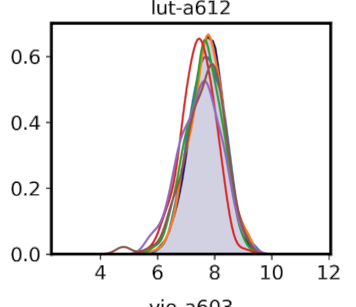

vio-a603

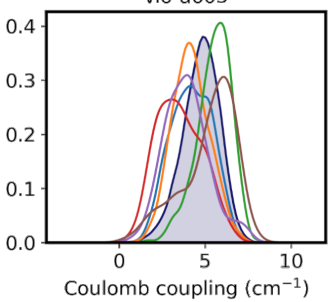

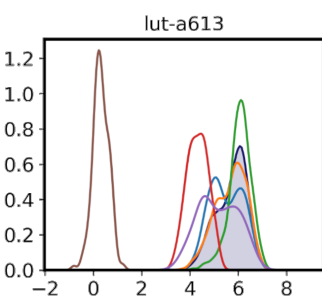

b

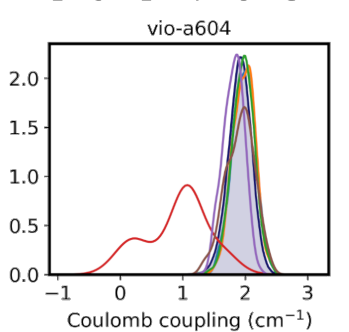

C
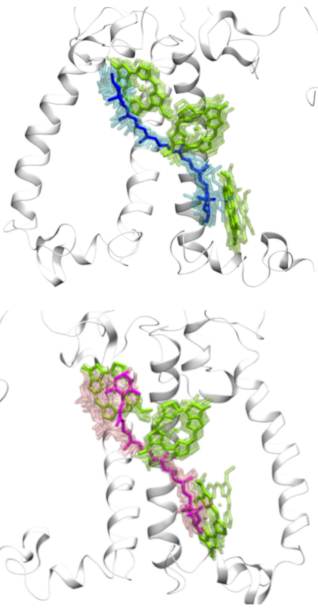

vio-a603

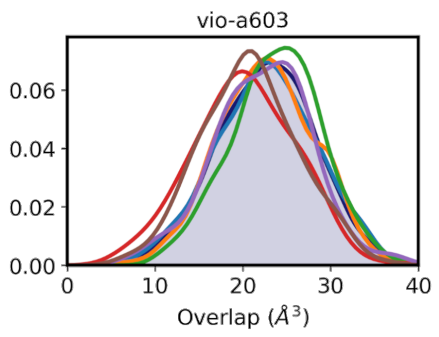

Figure 3: Coulomb coupling and overlap. The distributions are shown for both the PT-WTE and the $\mathrm{cMD}_{\text {CryoEM }}$ simulations, and the density of the $\mathrm{CMD}_{\text {CryoEM }}$ is shaded for an easier visualization. (a) Distributions of the Coulomb coupling between Lut and Chl a610, a612, a613 (L1) and between Vio and Chl a603, a603, a604 (L2). (b,c) fluctuations of the Car-Chl pairs shown in (a), for the L1 site (b) and the L2 site (c). (d) Distributions of the overlaps for the pair Lut-a612 (L1) and Vio-a603 (L2).

the electronic coupling is commonly approximated as the Coulomb interaction among transition densities of the pigments ${ }^{28,52}$, which can be projected onto atomic charges ${ }^{53}$. However, we note that the electronic coupling also contains short-range terms ${ }^{54-56}$, which are important for EET involving dark states such as the $S_{1}$ state of Cars. The short-range terms are difficult to compute, as they involve charge-transfer configurations ${ }^{57}$. In order to partly capture the short-range character of the Car-Chl interactions, we adopt a geometrical approximation of the electronic overlap in terms of rigid spheres centered on the atoms of the Car-Chl pair. In a previous study on CP29 ${ }^{58}$ we showed that the triplet-triplet Car-Chl electronic couplings, which contain only short-range terms, are strongly sensitive to this overlap parameter. We further note that this approximation has proven successful in modeling the CT mechanism of quenching in LHCII ${ }^{14}$. More details on the calculations of the coupling and the relation of the overlap parameter to short-range effects are provided in the SI.

First, we focus the analysis on the chlorophylls with the strongest coupling with the carotenoids in L1 and L2, namely Chl a612 with Lut and Chl a603 with Vio in L2. These chlorophylls lie at the interface between the external (with LHCII) and internal (with CP47) parts of PSII ${ }^{59}$, and belong to two groups of strongly coupled chlorophylls (a610-a611-a612 and a603-a609) that have been proposed to form the lowest energy excitons in CP29 ${ }^{29}$, and thus have been related to quenching. As shown in Figure 3a, our simulations indicate a remarkable insensitivity of the Coulomb coupling Lut-a612 to alterations of the L1 binding pocket, with the corresponding distributions all very similar and comparable with the $\mathrm{cMD}_{\mathrm{CryoEM}}$, in agreement with recent work ${ }^{39,60}$. On the other 
hand, the Coulomb coupling of Vio-a603 (Figure 3a) appears to be quite more variable, with the distributions in each cluster covering a wide energy range. Variations of this coupling are due to the high conformational freedom of the N-terminal domain, which interacts with the stromal ring of Vio, inducing large amplitude motions of the stromal side of this carotenoid within L2 (Figure 3c). Regarding the short-range interactions for both Car-Chl pairs, we note that the overlap

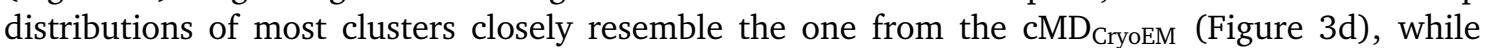
clusters 4 and 6 show some small deviations. As suggested by our previous work ${ }^{58}$, the short-range contribution has a steep dependence on the overlap parameter (See SI), and a $10 \AA^{3}$ reduction of the overlap is related to almost an order-of-magnitude drop in the short-range coupling. Thus, the differences observed in Figure 3d could give rise to significant variations in the total coupling. This observation, combined with the insensitivity of the Coulomb interaction observed in L1 (Figure 3a), suggests that a putative Lut- $a 612$ quenching channel can only be modulated by controlling the short-range coupling, rather than by altering the Coulomb coupling.

For both sites, we observe larger differences in the coupling distributions when considering the chlorophylls at the two extrema of Lut (Chl a610 and a613) and Vio (Chl a602 and a603) (Figure 3a), indicating that Car-Chl interactions on the two sides of the carotenoids are easier to tune than for the central Chl, thereby suggesting the presence of multiple Car-Chl quenching channels. In particular, Chl a613, which is anchored to helix A via Q214, shows the largest coupling displacement especially in clusters 4 and 6 (Figure 2d), indicating that the mobility on the lumenal side of the protein particularly affects this Chl.

Variations in the overlap are also found for Vio-a603 (Figure 3d), indicating that short range interactions are likely to play a role also for this Car-Chl pair. Still, the short range contributions for Vio-a603 are likely less impactful than for Lut-a612, as the increased conformational freedom of Vio in L2 (Figure 2d, Figure 3c) allows for sensible variations of its Coulomb coupling, as opposed to Lut (Figure 3a).

\section{A metastable 'open' conformation is observed in the lumenal side of CP29}

The above analysis indicates that conformational changes in CP29 can affect the geometry of the L1 and L2 sites. Still, it seems that altering the L1 site has almost no effect on the Lut-a612 Coulomb coupling. This implies that, without taking the short range contributions of the coupling and the modulation of the site energies into account, it is not possible to modulate the interactions of the Lut-a612 pair, despite extensive work suggesting that a EET quenching channel at the level of this pair is indeed present ${ }^{19,26}$. It is then interesting to investigate if, by enhancing the sampling of more distorted structures of CP29, it is possible to observe a variation in the Lut-a612 coupling, thereby indicating that major distortions of the protein can effectively alter the Car-Chl interactions at the level of their Coulomb coupling. To this end, we follow Refs ${ }^{46,61,62}$ where it was proposed that an alteration of the inter-helical crossing angle between helices A and B (the torsion defined by the nodes A2-A1-B1-B2 in Figure 4a) could account for NPQ in LHCII monomers. In addition, given the enhanced plasticity observed on the lumenal side of CP29, we have split the P1 angle of Ref ${ }^{61}$ in a lumenal P1 $\left(\mathrm{P} 1_{l}\right)$ and a stromal $\mathrm{P} 1\left(\mathrm{P} 1_{s}\right)$, so as to better describe the two sides of the complex (Figure 4a). The sampling over $\mathrm{P} 1_{l}$ and $\mathrm{P} 1_{s}$ is enhanced with well-tempered metadynamics ${ }^{63}$ in its multiple walkers ${ }^{64}$ variant, and successively refined with well-tempered metadynamics on path $\mathrm{CVs}^{65}$. Details on the simulation protocols are provided in the SI.

By enhancing the sampling over $\mathrm{P} 1_{l}$ and $\mathrm{P} 1_{s}$ an additional basin appears as a metastable state in the free energy surface (Figure $4 \mathrm{~b}$ ). This CP29 conformation differs significantly from the cryo$\mathrm{EM}^{41}$ structure and from the conformations sampled in the cMD ${ }_{\text {CryoEM }}$ and PT-WTE simulations, in particular for a wider inter-helical angle on the lumenal side (Figure 4c) of the protein. For this reason, we refer to this conformation as Open. The metastability of this conformation has been further confirmed with two $2 \mu \mathrm{s}$ long unbiased simulations, to which we refer collectively as $\mathrm{cMD}_{\text {Open }}$. We estimate that this conformation is separated by several $\mathrm{kcal} / \mathrm{mol}$ from the cryo-EM one (Figure 4b, Figure 9), and additional stabilizing factors would then be required to make it 

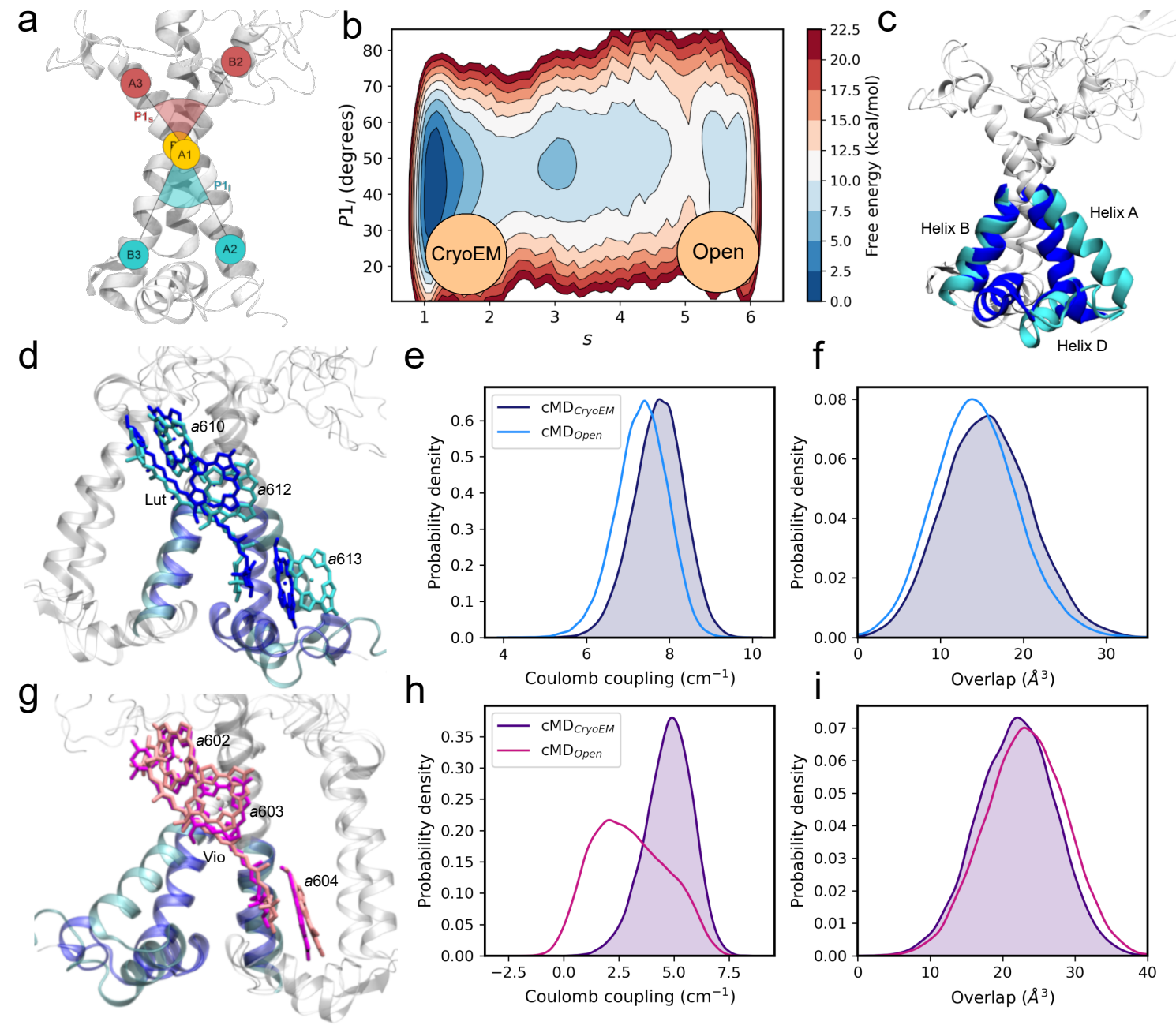

Figure 4: Metadynamics simulation and Open structure of CP29. (a) Definition of $\mathrm{P} 1_{l}$ and $\mathrm{P} 1_{s}$. (b) Free energy surface in the space of the path $\mathrm{CV} s$ and $\mathrm{P} 1_{l}$. The cryo-EM basin corresponds to small values of $s$, while the Open structure corresponds to values of $s$ comprised between 5 and 6 . (c) Comparison of the cryo-EM structure (PDB: 3JCU, dark blue) and the Open structure (cyan). (d) Alteration of the L1 pocket when moving from the cryo-EM structure (dark blue) to the Open structure (cyan). (e) Coulomb coupling and (f) Overlap for the Lut-a612 pair. (g) Alteration of the L2 pocket when moving from the cryo-EM structure (magenta) to the Open structure (pink). (h) Coulomb coupling and (i) Overlap for the Vio-a603 pair.

accessible at room temperature.

The wider $\mathrm{P} 1_{l}$ angle observed in Open is obtained by a large amplitude motion of the lumenal side of helix A, which additionally drags helix D away from helix B (Figure 4c). This conformational change has a direct influence over the L1 site, which appears to be significantly altered (Figure 4d). In particular, we find both a different position for the chlorophylls in the L1 site and a distorted geometry for Lutein, which keeps its interactions with helix D despite the departure of the latter. Finally, a softer effect is found for the L2 site (Figure $4 \mathrm{~g}$ ), due to the greater stiffness of helix B compared to helix A.

Given the pronounced alterations of the L1 site, it is natural to expect a variation in the Luta612 interactions in the Open conformation. However, we again observe a remarkable insensitivity of the Coulomb coupling, which is lowered by a very small $(\sim 7 \%)$ extent in the Open conformation 
(Figure 4e). It appears that even large rearrangements of the protein scaffold are not sufficient to modulate the Lut-a612 Coulomb interactions, suggesting that this Car-Chl interaction has little weight on the modulation of NPQ.

Instead, in agreement with the PT-WTE results (Figure 3a), the Vio-a603 coupling is quite variable (Figure 4h) due to the high amplitude motions of the N-terminal domain of CP29. Variations in the Coulomb coupling are also found for the chlorophylls on the two extrema of the carotenoids (Chls a610, a613 for Lut and Chl a602 for Vio, Figure 10), with the coupling of Lut-a613 being the most affected, due to the remarkable plasticity of helix A (Figure 4a). As noted before for the PT-WTE simulation, it appears that the main role of the protein scaffold dynamics is to alter the carotenoid geometry (Figure 4d,g), with direct consequences on its electronic structure and excited state properties. Finally, we note that the Open conformation has an effect also on the overlap distribution of Lut-a612 (Figure 4f), which decreases with respect to the PT-WTE results. Also in this case, short range interactions, very sensitive to the overlap between Car and Chl, can indeed be strongly tuned by the conformational change.

\section{Conclusions}

We have investigated the regulation of NPQ activity in the minor LHC of higher plants and green algae, CP29, through a fully atomistic computational approach which integrates molecular dynamics, enhanced sampling techniques, and the calculation of the electronic parameters connected with the quenching mechanisms.

By using enhanced sampling techniques, we have been able to explore the conformational space of CP29, identifying various conformers differing in their lumenal arrangement. We have shown that these conformers correspond to different arrangements of the L1 and L2 sites both in terms of the internal geometry of the Cars and the Car-Chl relative arrangements.

Notwithstanding the structural flexibility of sites L1 and L2, the interactions of the Cars with the central chlorophylls, $a 603$ and $a 612$, do not show very large variations. In particular, the coupling of the most coupled pair, Lut-a612, is surprisingly insensitive to protein conformational changes even when largely altered structures of CP29 are considered. These results seem to indicate that the use of the Coulomb component of the coupling, as commonly done in NPQ studies $28,39,52,61,66,67$ to characterize Chl-Car EET processes, is not sufficient. Two additional aspects must be included in the modeling, namely, the short-range effects, which become important for the closely-associated Chl-Car pairs, and the Car's internal structure, which is more strongly affected by protein conformation. In particular, since the electronic properties of the Cars are extremely sensitive to geometrical distortion, control of the Car geometry is one way for the protein to tune the quenching efficiency ${ }^{45}$, without significantly altering the Chl network. Finally, our results indicate that the couplings with external chlorophylls of sites L1 and L2 are more sensitive to protein conformational changes. This suggests that multiple Car-Chl EET quenching channels are active, and can be regulated by different protein motions. This underlines the importance of considering all the Chl-Car interactions in L1 and L2 sites to understand the excitation quenching in antenna complexes.

The motions observed in the CP29 complex isolated from the rest of PSII are still observed in the full complex, as shown by the electron density of the complex reconstructed by cryo-EM. Thus, the connection here revealed between the protein conformation, the structure of the pigment aggregate, and the quenching mechanisms specific to the complex per se are expected to be valid in the context of the PSII complex. The metastable 'open' conformation sampled is predicted by our simulations to be higher in energy in the isolated CP29 complex. Still, the conditions used for the present simulation and for most in vitro studies are different from those existing in vivo. The external triggers for NPQ activation, such as the proton gradient across the thylakoid membrane and the interaction with PsbS, are not present. Those triggers might well stabilize the 'open' conformation, for instance by promoting protein-protein interactions. ${ }^{68-70}$. What is more, due to structural similarity and sequence homology, the mechanisms proposed here are also relevant 
for other light-harvesting complexes and thus the atomistic details here revealed can represent a starting point for the design of complexes with optimized photoprotective functionalities.

\section{Computational Methods}

Details on the unbiased trajectory for CP29 started from the cryo-EM ( $\mathrm{cMD}_{\text {CryoEM }}$ ) and from the Open ( $\mathrm{cMD}_{\mathrm{Open}}$ ), the setup of the PT-WTE simulation, and the well-tempered metadynamics protocols are provided in the SI. Additional details on the dimensionality reduction, the clustering of the conformations in the PT-WTE simulation, the Coulomb coupling calculation and the role of the Overlap in describing the Charge-Transfer quenching are also provided.

\section{Conflicts of interest}

There are no conflicts to declare.

\section{Acknowledgments}

E.C., L.C. and B.M. acknowledge funding by the European Research Council, under the grant ERCAdG-786714 (LIFETimeS). M.L. acknowledges funding by University of Pisa under the grant BIHO. B.M. acknowledges financial support from MIUR through the PRIN 2017 (grant 201795SBA3_002). F.L.G. and S.A.G. acknowledge PRACE and the CSCS (project s1046) for supercomputing time.

\section{References}

[1] Croce, R. \& Van Amerongen, H. Natural strategies for photosynthetic light harvesting. Nature Chemical Biology 10, 492-501 (2014).

[2] Pinnola, A. \& Bassi, R. Molecular mechanisms involved in plant photoprotection. Biochemical Society Transactions 46, 467-482 (2018).

[3] Müller, P., Li, X. P. \& Niyogi, K. K. Non-photochemical quenching. a response to excess light energy. Plant Physiology 125, 1558-1566 (2001).

[4] Pascal, A. A. et al. Molecular basis of photoprotection and control of photosynthetic lightharvesting. Nature 436, 134-137 (2005).

[5] Li, Z., Wakao, S., Fischer, B. B. \& Niyogi, K. K. Sensing and responding to excess light. Annual Review of Plant Biology 60, 239-260 (2009).

[6] Ruban, A. V., Johnson, M. P. \& Duffy, C. D. The photoprotective molecular switch in the photosystem ii antenna. Biochimica et Biophysica Acta - Bioenergetics 1817, 167-181 (2012).

[7] Niyogi, K. K. \& Truong, T. B. Evolution of flexible non-photochemical quenching mechanisms that regulate light harvesting in oxygenic photosynthesis. Current Opinion in Plant Biology 16, 307-314 (2013).

[8] Nicol, L., Nawrocki, W. J. \& Croce, R. Disentangling the sites of non-photochemical quenching in vascular plants. Nature Plants 5, 1177-1183 (2019).

[9] Ruban, A. V. et al. Identification of a mechanism of photoprotective energy dissipation in higher plants. Nature 450, 575-578 (2007). 
[10] Ahn, T. K. et al. Architecture of a charge-transfer state regulating light harvesting in a plant antenna protein. Science 320, 794-797 (2008).

[11] Dall'Osto, L. et al. Two mechanisms for dissipation of excess light in monomeric and trimeric light-harvesting complexes. Nat. Plants 3, 17033 (2017).

[12] Bode, S. et al. On the regulation of photosynthesis by excitonic interactions between carotenoids and chlorophylls. Proceedings of the National Academy of Sciences of the United States of America 106, 12311-12316 (2009).

[13] Holt, N. E. et al. Carotenoid cation formation and the regulation of photosynthetic light harvesting. Science 307, 433-436 (2005).

[14] Cupellini, L., Calvani, D., Jacquemin, D. \& Mennucci, B. Charge transfer from the carotenoid can quench chlorophyll excitation in antenna complexes of plants. Nature Communications 11, 662 (2020).

[15] Krüger, T. P. et al. Controlled disorder in plant light-harvesting complex ii explains its photoprotective role. Biophys. J. 102, 2669-2676 (2012).

[16] Krüger, T. P., Ilioaia, C., Johnson, M. P., Ruban, A. V. \& van Grondelle, R. Disentangling the low-energy states of the major light-harvesting complex of plants and their role in photoprotection. Biochim. Biophys. Acta - Bioenerg. 1837, 1027-1038 (2014).

[17] Schlau-Cohen, G. S. et al. Single-molecule identification of quenched and unquenched states of lhcii. J. Phys. Chem. Lett. 6, 860-867 (2015).

[18] Kondo, T. et al. Microsecond and millisecond dynamics in the photosynthetic protein lhcsr1 observed by single-molecule correlation spectroscopy. Proc. Natl. Acad. Sci. 116, 1124711252 (2019).

[19] Mascoli, V. et al. Capturing the Quenching Mechanism of Light-Harvesting Complexes of Plants by Zooming in on the Ensemble. Chem 5, 1-13 (2019).

[20] Chmeliov, J. et al. The nature of self-regulation in photosynthetic light-harvesting antenna. Nature Plants 2, 16045 (2016).

[21] Mascoli, V., Gelzinis, A., Chmeliov, J., Valkunas, L. \& Croce, R. Light-harvesting complexes access analogue emissive states in different environments. Chem. Sci. 11, 5697-5709 (2020).

[22] Nicol, L. \& Croce, R. The psbs protein and low ph are necessary and sufficient to induce quenching in the light-harvesting complex of plants lhcii. Scientific Reports 11 (2021).

[23] Miloslavina, Y., de Bianchi, S., Dall'Osto, L., Bassi, R. \& Holzwarth, A. R. Quenching in arabidopsis thaliana mutants lacking monomeric antenna proteins of photosystem ii. J. Biol. Chem. 286, 36830-36840 (2011).

[24] Pan, X. et al. Structural insights into energy regulation of light-harvesting complex cp29 from spinach. Nature Struct. Mol. Biol. 18, 309-15 (2011).

[25] Pan, X., Liu, Z., Li, M. \& Chang, W. Architecture and function of plant light-harvesting complexes ii. Curr. Opin. Struct. Biol. 23, 515-25 (2013).

[26] Gacek, D. A. et al. Carotenoid dark state to chlorophyll energy transfer in isolated lightharvesting complexes cp24 and cp29. Photosynthesis Research 96, 1-12 (2019).

[27] Guardini, Z., Bressan, M., Caferri, R., Bassi, R. \& Dall'Osto, L. Identification of a pigment cluster catalysing fast photoprotective quenching response in cp29. Nature Plants 6, 303313 (2020). 
[28] Fox, K. F. et al. A possible molecular basis for photoprotection in the minor antenna proteins of plants. Biochimica et Biophysica Acta - Bioenergetics 1859, 471-481 (2018).

[29] Mascoli, V., Novoderezhkin, V., Liguori, N., Xu, P. \& Croce, R. Design principles of solar light harvesting in plants: Functional architecture of the monomeric antenna CP29. Biochimica et Biophysica Acta (BBA) - Bioenergetics 1861, 148156 (2020). URL https://doi .org/10.1016\%2Fj. bbabio.2020.148156.

[30] Laio, A. \& Gervasio, F. L. Metadynamics: a method to simulate rare events and reconstruct the free energy in biophysics, chemistry and material science. Reports on Progress in Physics 71, 126601 (2008).

[31] Bussi, G. \& Laio, A. Using metadynamics to explore complex free-energy landscapes. Nature Reviews Physics 2, 200-212 (2020).

[32] Liguori, N., Croce, R., Marrink, S. J. \& Thallmair, S. Molecular dynamics simulations in photosynthesis. Photosynthesis Research 144, 273-295 (2020).

[33] Cupellini, L., Bondanza, M., Nottoli, M. \& Mennucci, B. Successes \& challenges in the atomistic modeling of light-harvesting and its photoregulation. BBA-Bioenergetics 1861, 148049 (2020).

[34] Bonomi, M. \& Parrinello, M. Enhanced sampling in the well-tempered ensemble. Phys. Rev. Lett. 104 (2010). URL https://doi .org/10.1103\%2Fphysrevlett.104. 190601.

[35] Deighan, M., Bonomi, M. \& Pfaendtner, J. Efficient simulation of explicitly solvated proteins in the well-tempered ensemble. J. Chem. Theory Comput. 8, 2189-2192 (2012). URL https://doi.org/10.1021\%2Fct300297t.

[36] Mattedi, G., Acosta-Gutiérrez, S., Clark, T. \& Gervasio, F. L. A combined activation mechanism for the glucagon receptor. Proc Natl Acad Sci USA 117, 15414-15422 (2020). URL https://doi.org/10.1073\%2Fpnas. 1921851117.

[37] Sutto, L. \& Gervasio, F. L. Effects of oncogenic mutations on the conformational free-energy landscape of EGFR kinase. Proceedings of the National Academy of Sciences 110, 10616-10621 (2013). URL https://doi.org/10.1073/pnas.1221953110.

[38] Altis, A., Nguyen, P. H., Hegger, R. \& Stock, G. Dihedral angle principal component analysis of molecular dynamics simulations. The Journal of Chemical Physics 126, 244111 (2007). URL https://doi .org/10.1063\%2F1.2746330.

[39] Lapillo, M., Cignoni, E., Cupellini, L. \& Mennucci, B. The energy transfer model of nonphotochemical quenching: Lessons from the minor CP29 antenna complex of plants. Biochimica et Biophysica Acta (BBA) - Bioenergetics 1861, 148282 (2020).

[40] Kavalenka, A. A. et al. Site-directed spin-labeling study of the lightharvesting complex CP29. Biophysical Journal 96, 3620-3628 (2009). URL https://doi.org/10.1016/j.bpj. 2009.01.038.

[41] Wei, X. et al. Structure of spinach photosystem II-LHCII supercomplex at 3.2-AA resolution. Nature 534, 69-74 (2016).

[42] Sheng, X. et al. Structural insight into light harvesting for photosystem II in green algae. Nat. Plants 5, 1320-1330 (2019). URL https://doi.org/10.1038\%2Fs41477-019-0543-4.

[43] Liguori, N., Roy, L. M., Opacic, M., Durand, G. \& Croce, R. Regulation of light harvesting in the green alga chlamydomonas reinhardtii: The c-terminus of LHCSR is the knob of a dimmer switch. J. Am. Chem. Soc. 135, 18339-18342 (2013). URL https://doi.org/10.1021\%2Fja4107463. 
[44] Ioannidis, N. E., Papadatos, S. \& Daskalakis, V. Energizing the light harvesting antenna: Insight from CP29. Biochimica et Biophysica Acta (BBA) - Bioenergetics 1857, 1643-1650 (2016). URL https://doi .org/10.1016\%2Fj . bbabio. 2016.07.005.

[45] Liguori, N. et al. Different carotenoid conformations have distinct functions in lightharvesting regulation in plants. Nature Communications 8, 1-9 (2017).

[46] Li, H. et al. Dynamical and allosteric regulation of photoprotection in light harvesting complex ii. Sci. China Chem. 63, 1121-1133 (2020).

[47] Saccon, F., Durchan, M., Polívka, T. \& Ruban, A. V. The robustness of the terminal emitter site in major LHCII complexes controls xanthophyll function during photoprotection. Photochemical and Photobiological Sciences 19, 1308-1318 (2020).

[48] Son, M., Pinnola, A., Gordon, S. C., Bassi, R. \& Schlau-Cohen, G. S. Observation of dissipative chlorophyll-to-carotenoid energy transfer in light-harvesting complex ii in membrane nanodiscs. Nature Communications 11 (2020).

[49] Saccon, F. et al. A protein environment-modulated energy dissipation channel in LHCII antenna complex. iScience 23, 101430 (2020). URL https://doi.org/10.1016\%2Fj.isci.2020.101430.

[50] Llansola-Portoles, M. J. et al. Twisting a $\beta$-carotene, an adaptive trick from nature for dissipating energy during photoprotection. Journal of Biological Chemistry 292, 1396-1403 (2017). URL https://doi.org/10.1074\%2Fjbc.m116.753723.

[51] Polívka, T. \& Sundström, V. Dark excited states of carotenoids: Consensus and controversy. Chemical Physics Letters 477, 1-11 (2009). URL https://doi.org/10.1016\%2Fj.cplett.2009.06.011.

[52] Balevičius, V. et al. Fine control of chlorophyll-carotenoid interactions defines the functionality of light-harvesting proteins in plants. Scientific Reports 7, 1-10 (2017).

[53] Madjet, M. E., Abdurahman, A. \& Renger, T. Intermolecular Coulomb Couplings from Ab Initio Electrostatic Potentials: Application to Optical Transitions of Strongly Coupled Pigments in Photosynthetic Antennae and Reaction Centers. Journal of Physical Chemistry B 110, 17268-17281 (2006).

[54] Cupellini, L., Corbella, M., Mennucci, B. \& Curutchet, C. Electronic energy transfer in biomacromolecules. WIREs Comput Mol Sci Computational Molecular Science 9 (2018). URL https://doi.org/10.1002\%2Fwcms . 1392.

[55] Hsu, C.-p., You, Z.-Q. \& Chen, H.-C. Characterization of the Short-Range Couplings in Excitation Energy Transfer. J. Phys. Chem. C 112, 1204-1212 (2008). URL http://pubs.acs.org/cgi-bin/doilookup/?10.1021/jp076512i.

[56] Hsu, C.-p. The electronic couplings in electron transfer and excitation energy transfer. Acc. Chem. Res. 42, 509-18 (2009).

[57] You, Z.-Q. \& Hsu, C.-P. Theory and calculation for the electronic coupling in excitation energy transfer. Int. J. Quantum Chem. 114, 102-115 (2014).

[58] Cupellini, L., Jurinovich, S., Prandi, I. G., Caprasecca, S. \& Mennucci, B. Photoprotection and triplet energy transfer in higher plants: the role of electronic and nuclear fluctuations. Phys. Chem. Chem. Phys. 18, 11288-11296 (2016).

[59] Xu, P., Roy, L. M. \& Croce, R. Functional organization of photosystem II antenna complexes: CP29 under the spotlight. Biochimica et Biophysica Acta (BBA) - Bioenergetics 1858, 815-822 (2017). URL https://doi .org/10.1016\%2Fj . bbabio.2017.07.003. 
[60] Maity, S., Daskalakis, V., Elstner, M. \& Kleinekathöfer, U. Multiscale qm/mm molecular dynamics simulations of the trimeric major light-harvesting complex ii. Physical Chemistry Chemical Physics 23, 7407-7417 (2021).

[61] Daskalakis, V. et al. Structural basis for allosteric regulation in the major antenna trimer of photosystem ii. Journal of Physical Chemistry B 123, 9609-9615 (2019).

[62] Daskalakis, V., Papadatos, S. \& Stergiannakos, T. The conformational phase space of the photoprotective switch in the major Light Harvesting Complex II. Chemical Communications 56, 11215-11218 (2020).

[63] Barducci, A., Bussi, G. \& Parrinello, M. Well-Tempered Metadynamics: A Smoothly Converging and Tunable Free-Energy Method. Physical Review Letters 100, 1-4 (2008).

[64] Raiteri, P., Laio, A., Gervasio, F. L., Micheletti, C. \& Parrinello, M. Efficient reconstruction of complex free energy landscapes by multiple walkers metadynamics. Journal of Physical Chemistry B 110, 3533-3539 (2006).

[65] Branduardi, D., Gervasio, F. L. \& Parrinello, M. From a to b in free energy space. The Journal of Chemical Physics 126, 054103 (2007). URL https://doi.org/10.1063\%2F1. 2432340.

[66] Chmeliov, J. et al. An 'all pigment' model of excitation quenching in LHCII. Physical Chemistry Chemical Physics 17, 15857-15867 (2015).

[67] Fox, K. F. et al. The carotenoid pathway: what is important for excitation quenching in plant antenna complexes? Physical Chemistry Chemical Physics 19, 22957-22968 (2017).

[68] Ort, D. R. et al. Redesigning photosynthesis to sustainably meet global food and bioenergy demand. Proceedings of the National Academy of Sciences 112, 8529-8536 (2015).

[69] Kromdijk, J. et al. Improving photosynthesis and crop productivity by accelerating recovery from photoprotection. Science 354, 857-861 (2016).

[70] Garcia-Molina, A. \& Leister, D. Accelerated relaxation of photoprotection impairs biomass accumulation in arabidopsis. Nature Plants 6, 9-12 (2020). 